\title{
Standard coagulation assays alone are not sufficient to exclude surgically relevant rivaroxaban plasma concentrations
}

\author{
Alexander Kaserer ${ }^{1 *} \mathbb{D}$, Andreas Schedler ${ }^{1}$, Burkhardt Seifert ${ }^{2}$, Donat R. Spahn ${ }^{1}$, Jan-Dirk Studt ${ }^{3}$ and Philipp Stein ${ }^{1}$
}

\begin{abstract}
Background: While mainly larger hospitals have introduced routine anti-Xa assays for rivaroxaban (RXA), these are not readily available to smaller hospitals often relying on routine coagulation tests such as prothrombin time (PT) and activated partial thromboplastin time (aPTT).

The aim of our study was to investigate the effect of RXA plasma concentration on the standard coagulation tests PT (Quick test and INR) and aPTT in a large group of real-life patients. We further assessed whether normal results of these standard coagulation assays are sufficient to exclude surgically relevant RXA plasma concentration, defined as $>50 \mathrm{mcg} / \mathrm{l}$.

Methods: This retrospective study included all patients between 2012 and 2016 where anti-Xa (calibrated for RXA), PT (Quick test and INR), and/or aPTT were determined from the same sample. PT is expressed as Quick value (\% of normal plasma pool). In total, 1027 measurements in 622 patients were eligible for analysis: 752 measurements of 505 patients for Quick/INR and 594 measurements of 417 patients for aPTT.

Results: A moderate correlation of PT/Quick (Pearson's correlation coefficient $-0.59 ; p<0.001$ ), INR (Pearson's correlation coefficient $0.5 ; p<0.001$ ), and aPTT (Pearson's correlation coefficient $0.53 ; p<0.001$ ) with RXA plasma concentration was observed. However, in 50\% of all samples with a normal PT/Quick, in 25\% of all samples with a normal INR and in $80 \%$ of all samples with a normal aPTT residual RXA plasma concentration was surgically relevant.
\end{abstract}

Conclusion: Although a moderate correlation of RXA plasma concentration with PT/Quick, INR, and aPTT was observed, standard coagulation assays are not sufficient to exclude surgically relevant RXA plasma concentrations.

Keywords: Anticoagulation, Rivaroxaban, Coagulation assays, Surgery

\section{Background}

Rivaroxaban (RXA) is a direct oral anticoagulant (DOAC) which inhibits factor Xa (Xa). It is widely used for thromboprophylaxis in orthopaedic surgery, prevention of systemic embolism in non-valvular atrial fibrillation, and treatment and secondary prevention of venous thromboembolism (Swissmedicinfo.ch 2017). In many patients admitted for elective and non-elective surgery, a surgically relevant residual RXA plasma concentration is observed, defined as $>50 \mathrm{mcg} / \mathrm{l}$. This is

\footnotetext{
* Correspondence: alexander.kaserer@usz.ch

${ }^{1}$ Institute of Anaesthesiology, University and University Hospital Zurich,

Raemistrasse 100, 8091 Zurich, Switzerland

Full list of author information is available at the end of the article
}

especially important in an emergency setting (e.g., trauma) since it may affect patient management or be prohibitive for surgery.

For determining RXA plasma concentration, highperformance liquid chromatography-mass spectrometry (HPLC-MS) is considered the gold standard (Asmis et al. 2012). However, this method is time-consuming, expensive, and therefore not suitable for routine clinical use. In comparison, chromogenic anti-Xa assays calibrated for RXA are fast, affordable, and show a very good agreement with HPLC-MS (Studt et al. 2017). While most university hospitals or other large hospitals have introduced anti-Xa assays for RXA, these are not readily available to smaller hospitals often relying on

(C) The Author(s). 2019 Open Access This article is distributed under the terms of the Creative Commons Attribution 4.0 International License (http://creativecommons.org/licenses/by/4.0/), which permits unrestricted use, distribution, and 
routine coagulation tests such as prothrombin time (PT) and activated partial thromboplastin time (aPTT). In German-speaking countries, a plasma sample's tissue factor-induced coagulation time is typically expressed as Quick value (\%) in relation to that of a normal plasma pool; the longer the PT, the lower the Quick value (\%).

The aim of our study was to investigate the effect of RXA plasma concentration as determined by anti-Xa assay on the standard coagulation tests PT (Quick test and INR) and aPTT. Further, we assessed whether normal values of these standard coagulation assays are sufficient to exclude surgically relevant RXA plasma concentration which was defined as $>50 \mathrm{mcg} / \mathrm{l}$ in a large group of real-life patients.

\section{Material and methods}

This study was approved by the local ethics committee (Kantonale Ethikkomission Zurich, Switzerland, KEKZH-No: 2017-00164).

\section{Study design}

This retrospective single-center study included all adult patients between 2012 and 2016 where anti-Xa (calibrated for RXA), PT (Quick test and INR), and/or aPTT were determined from the same plasma sample.

Patients with a RXA concentration below the assay's limit of quantification $(20 \mathrm{mcg} / \mathrm{l})$, without documented RXA intake, without sufficient documentation, or with concomitant treatment by heparin or other anticoagulants were excluded. In total, 1027 measurements in 622 patients were eligible for investigation (Fig. 1).

\section{Study goals}

Our study aimed to investigate the impact of anti-Xa determined RXA plasma concentration as determined by anti-Xa assay on the standard coagulation tests PT (Quick test and INR) and aPTT. Moreover, we assessed in a large group of real-life patients if normal values of these standard assays were sufficient to exclude a surgically relevant RXA plasma concentration.

\section{Sample preparation and coagulation assays}

Venous blood was drawn into tubes containing 0.109 M sodium citrate (BD Vacutainer, Plymouth, UK). Samples were transported immediately to the ISO 17025 accredited hemostasis laboratory of the University Hospital Zürich where all coagulation assays were performed.

RXA plasma concentration was determined by chromogenic anti-Xa assay calibrated for RXA (Studt et al. 2017). During the daytime, the DiXaI assay (Hyphen Biomed, Neuville-sur-Oise, France) was used due to its insensitivity to heparin and at all other times a routine anti-Xa assay (Biophen Heparin LRT, Hyphen Biomed) with the same set of calibrators. Regular internal and external quality control was performed for both assays, and their agreement was confirmed repetitively.

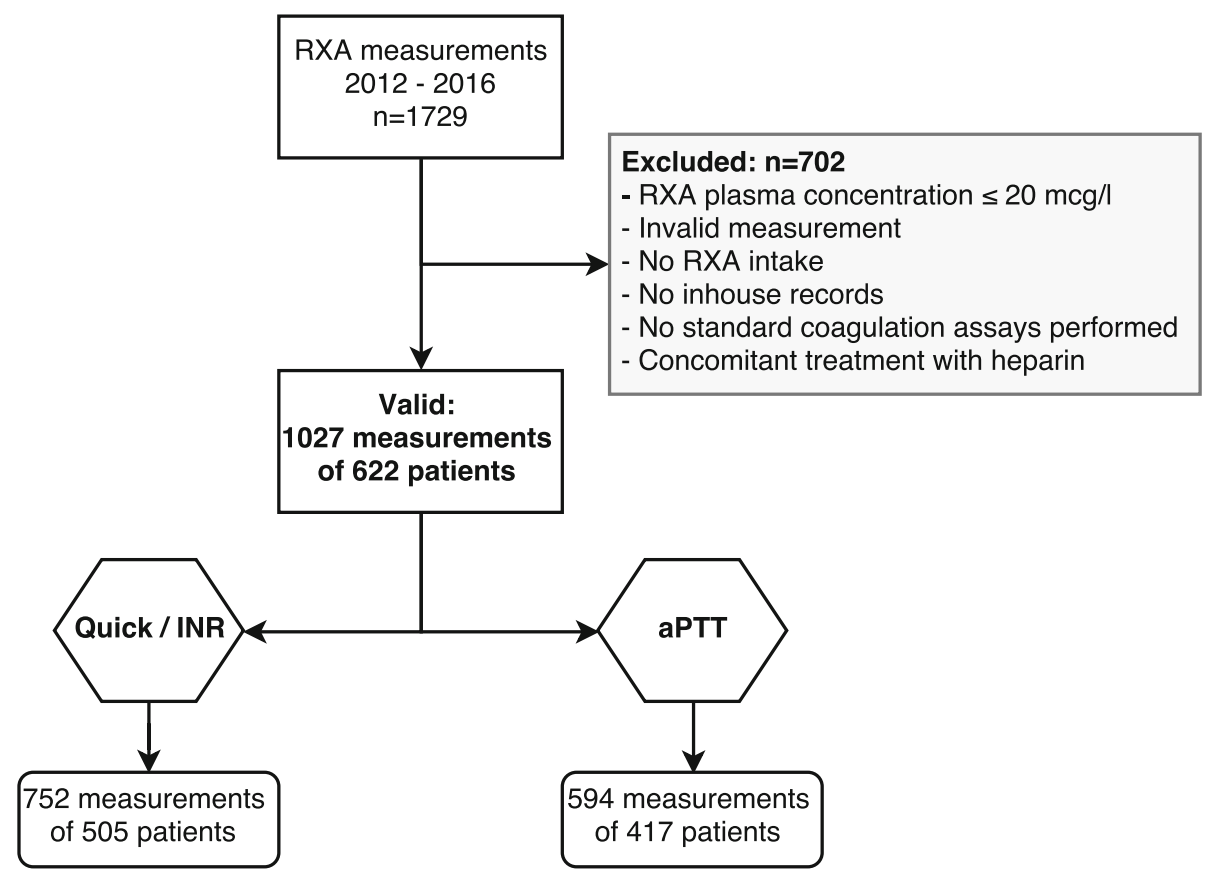

Fig. 1 Flowchart showing the number of included and excluded patients for each standard coagulation assay. Not all patients had a concomitant Quick/INR and aPTT measurement, explaining the different counts in the Quick/INR and aPTT groups. RXA = rivaroxaban, INR=international normalized ratio, aPTT = activated partial thromboplastin time, Quick = prothrombin time expressed as \% of the normal plasma pool 
In German-speaking countries, the tissue factor-induced coagulation time is traditionally expressed as Quick (\%) instead of PT (s); therefore, PT is presented here as Quick (\%) and INR. PT (Quick test) was determined using Innovin as thromboplastin reagent, and aPTT using the Actin FS reagent (both Siemens Healthcare, Marburg, Germany). Coagulometers were Siemens BCS XP and CS5100. Normal values for standard coagulation assays were Quick > 70\%, INR < 1.2, and aPTT 24-36 s.

\section{Variables and data collection}

Medical records were reviewed of all patients where RXA plasma concentration was determined between 2012 and 2016. Age, sex, indication for anticoagulation, RXA dosage, body mass index (BMI), creatinine serum level, and glomerular filtration rate (GFR) according to CKD-EPI formula were extracted from the hospital's database. The results of standard coagulation assays (Quick, INR, aPTT) originating from those samples in which RXA plasma concentration had been determined were extracted from the hospital's laboratory information system. Data were transferred to a spreadsheet for evaluation (Excel 2016, Microsoft Corporation, Redmond, USA).

\section{Statistical analyses}

Categorical data are reported as frequency $(n)$ and percent (\%) and numerical data as mean and standard deviation (SD) or median [IQR]. Due to the skew distribution of RXA plasma concentrations, INR and aPTT were logarithmically transformed. Correlation of RXA plasma concentrations with each of the standard coagulation assays was investigated using Pearson's correlation coefficient. A receiver operating characteristic (ROC) curve analysis was performed calculating the area under the curve (AUC) for each standard coagulation assay. Statistical significance was set as a two-tailed $p$ value of less than 0.05. All statistical analyses were performed with IBM SPSS Statistics (IBM SPSS Statistics v25.0., Armonk, NY: IBM Corp.).

\section{Results}

\section{Patient characteristics}

Patients were $58 \%$ male and $42 \%$ female, with a mean age of $69 \pm 16$ years. Renal function was mildly impaired with a mean GFR of $68 \pm 27 \mathrm{ml} / \mathrm{min}$ and mean serum creatinine of $103 \pm 63 \mathrm{mcmol} / \mathrm{l}$. Indications for anticoagulation included atrial fibrillation, pulmonary embolism, deep vein thrombosis, and thromboprophylaxis. The most frequent RXA dose was $20 \mathrm{mg} / \mathrm{day}$. The mean values of standard coagulation assays (PT/Quick, aPTT) were within the normal range (PT/Quick 70120\%; aPTT 24-36 s), and INR was slightly increased (Table 1).
Table 1 Overview

\begin{tabular}{|c|c|c|}
\hline \multicolumn{2}{|l|}{ Age (years), mean $\pm S D$} & $69 \pm 16$ \\
\hline \multicolumn{2}{|l|}{ Sex male, $n(\%)$} & $595(58 \%)$ \\
\hline \multicolumn{2}{|l|}{ Height $(\mathrm{cm})$, mean $\pm S D$} & $170 \pm 10$ \\
\hline \multicolumn{2}{|l|}{ Weight $(\mathrm{kg})$, mean $\pm \mathrm{SD}$} & $76 \pm 18$ \\
\hline \multicolumn{2}{|l|}{$\mathrm{BMI}\left(\mathrm{kg} / \mathrm{m}^{2}\right)$, mean $\pm \mathrm{SD}$} & $26 \pm 5$ \\
\hline \multicolumn{2}{|c|}{ Serum creatinine $(\mathrm{mmol} / \mathrm{l})$, mean $\pm \mathrm{SD}$} & $103 \pm 63$ \\
\hline \multicolumn{2}{|c|}{ GFR CKD-EPI (ml/min), mean \pm SD } & $68 \pm 27$ \\
\hline \multirow[t]{5}{*}{ Indication for RXA, n (\%) } & Atrial fibrillation & $574(56 \%)$ \\
\hline & Pulmonary embolism & $128(13 \%)$ \\
\hline & Thrombosis & $168(16 \%)$ \\
\hline & Prophylaxis & $83(8 \%)$ \\
\hline & Other & $74(7 \%)$ \\
\hline \multirow{5}{*}{$\begin{array}{l}\text { RXA dose (mg/day) } \\
(n=1020) \\
n(\%)\end{array}$} & 10 & $69(7 \%)$ \\
\hline & 15 & $200(20 \%)$ \\
\hline & 20 & $712(69 \%)$ \\
\hline & 30 & $38(4 \%)$ \\
\hline & 40 & $1(0 \%)$ \\
\hline \multicolumn{2}{|c|}{ Quick (\%), mean \pm SD; median [IQR] } & $76 \pm 23 ; 77[74-79]$ \\
\hline \multicolumn{2}{|c|}{ INR, mean $\pm \mathrm{SD}$, median [IQR] } & $1.3 \pm 0.6 ; 1.2[1.2-1.3]$ \\
\hline \multicolumn{2}{|c|}{$\mathrm{aP} T \mathrm{(s)}$, mean $\pm \mathrm{SD}$; median $[\mathrm{IQR}]$} & $30 \pm 12 ; 28[28-29]$ \\
\hline
\end{tabular}

\section{PT/Quick and INR}

Correlation of PT/Quick and RXA plasma concentration was moderate (Pearson's correlation coefficient $0.59, p<0.001$; Fig. 2). Nevertheless, in 50\% of all samples with a normal PT/Quick, the residual RXA plasma concentration was still elevated to a surgically relevant level $>50 \mathrm{mcg} / \mathrm{l}$, up to a maximum of $407 \mathrm{mcg} / \mathrm{l}$ (AUC: $0.74,95 \%$ CI 0.71 to $0.78, p<0.001$ ) (Table 2).

Similarly, the correlation of INR with RXA plasma concentration was only moderate (Pearson's correlation coefficient $0.5, p<0.001$; Fig. 3). Again, $25 \%$ of all patients with INR $<1.2$ had a surgically relevant residual RXA plasma concentration up to a maximum of $268 \mathrm{mcg} / \mathrm{l}$ (AUC $0.74,95 \%$ CI 0.70 to $0.77, p<0.001$ ) (Table 2).

aPTT

APTT showed as well a moderate correlation with RXA plasma concentration (Pearson's correlation coefficient $0.53, p<0.001$; Fig. 4). As many as $80 \%$ of all patients with a normal aPTT $(<36 \mathrm{~s})$ had a surgically relevant RXA plasma concentration up to a maximum of $437 \mathrm{mcg} / \mathrm{l}$ (AUC 0.75, 95\% CI 0.71 to $0.79, p<0.001$ ) (Table 2).

\section{Discussion}

An increasing number of patients admitted to the emergency department are anticoagulated with DOACs such as RXA. If such information is lacking-e.g., because of an unconscious patient-significant bleeding during 


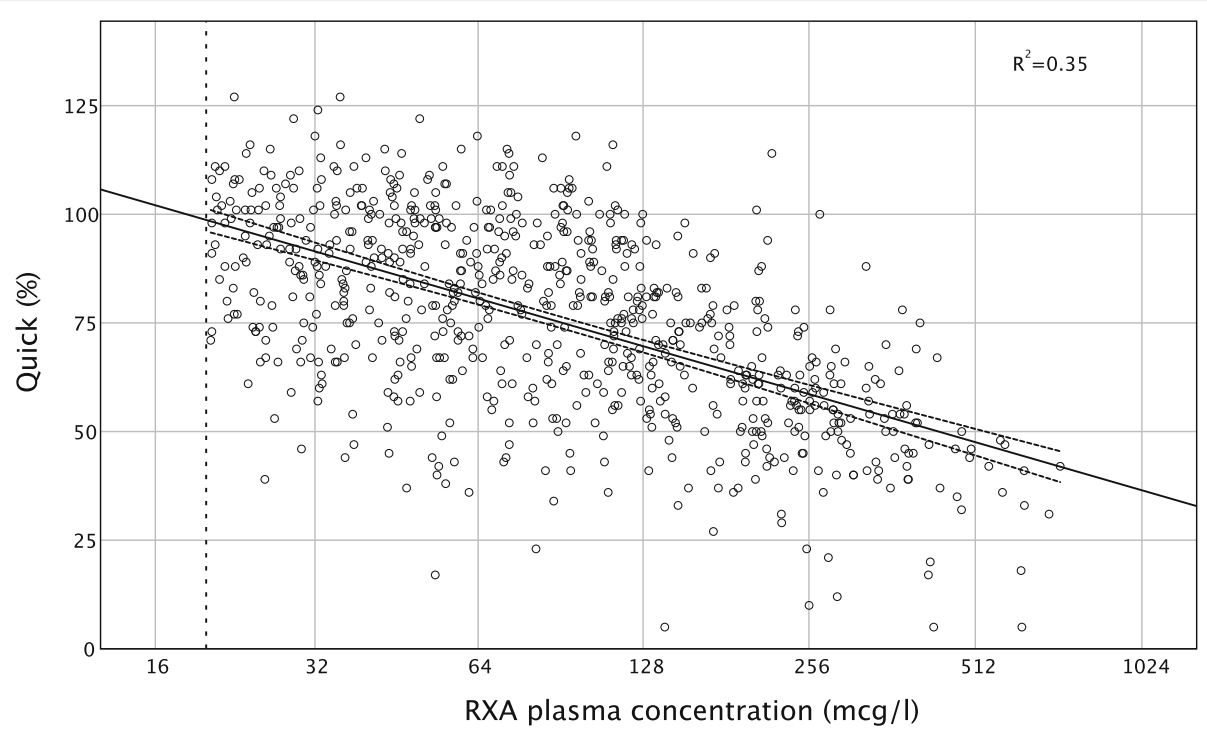

Fig. 2 Correlation of rivaroxaban plasma concentration and Quick. A statistically significant correlation is observed (Pearson's correlation coefficient $-0.59, p<0.001 ; R^{2}$ linear 0.35 ). Quick = prothrombin time expressed as $\%$ of normal plasma pool, RXA = rivaroxaban

invasive procedures or thrombolysis may be the consequence. Furthermore, factors were identified such as renal insufficiency or amiodarone co-medication that may result in a higher-than-expected residual RXA concentration even if standard preoperative interruption intervals are observed (Kaserer et al. 2018). Fast and reliable quantification of a DOAC's plasma concentration is therefore crucial for the clinical management. While many universities or other large hospitals have introduced routine anti-Xa assays for RXA, smaller hospitals often have to rely on standard coagulation assays such as PT/Quick, INR, or aPTT. Although we observed a statistically significant (but moderate) correlation of the results of these standard assays with RXA plasma concentration, these were not sufficient to exclude a surgically relevant residual RXA level.

In line with our results, a close correlation of anti-Xa activity, PT, and aPTT has been observed previously in healthy individuals (Turkoglu 2015). APTT showed a slight to moderate prolongation depending on the RXA concentration (Samama et al. 2010). In an in vitro study simulating RXA peak plasma concentrations in healthy volunteers, the aPTT was prolonged two-fold by RXA

Table 2 Patients with normal standard coagulation assays but residual $\mathrm{RXA}$ level $>50 \mathrm{mcg} / \mathrm{l}$

\begin{tabular}{|c|c|c|c|c|c|c|}
\hline & \multicolumn{2}{|c|}{$\mathrm{RXA}>50 \mathrm{mcg} / \mathrm{l}$} & \multicolumn{4}{|c|}{ RXA plasma concentration $\mathrm{mcg} / \mathrm{l}$} \\
\hline & $n$ & $\%$ & Mean & SD & Min & Max \\
\hline Quick > 70\% & 264 & $50 \%$ & 77 & 57 & 20.4 & 407 \\
\hline INR $<1.2$ & 133 & $25 \%$ & 63 & 42 & 20.5 & 268 \\
\hline $\mathrm{aPTT}<36 \mathrm{~s}$ & 333 & $80 \%$ & 100 & 79 & 20.4 & 437 \\
\hline
\end{tabular}

peak plasma levels of $389 \pm 106 \mathrm{mcg} / \mathrm{l}$ to $617 \pm 149 \mathrm{mcg} / \mathrm{l}$ (Hillarp et al. 2014). Moreover, Ikeda and Tachibana showed in patients receiving RXA for atrial fibrillation that aPTT tends to be prolonged (Ikeda and Tachibana 2016). The correlation of aPTT and RXA plasma level does not only depend on the latter since there is considerable variability among laboratories and various aPTT reagents (Samuelson et al. 2017; Samama et al. 2013). Although the reagent used in our laboratory (Actin FS, Siemens Healthcare) is comparatively sensitive to RXA (Samama et al. 2013), aPTT was not prolonged in $80 \%$ of patients with a residual RXA level $>50 \mathrm{mcg} / \mathrm{l}$.

The effect of RXA plasma level on aPTT was weaker than for PT/Quick and INR. It was shown, that RXA prolongs PT/Quick in a linear and concentration-dependent manner (Samama et al. 2010). Baglin et al. demonstrated that normal PT cannot exclude an anticoagulant effect of DOAC, but can indicate a subtherapeutic plasma level (Baglin 2013). Results vary according to the thromboplastin reagent (Samama et al. 2010; Dale et al. 2014). Innovin (Siemens Healtcare), which is used in our laboratory, has an intermediate sensitivity towards RXA as compared with other reagents, e.g., recombiplastin, neoplastin, or neoplastin plus (Samama et al. 2010). The RXA plasma concentration required to prolong the PT two-fold is $301 \mathrm{mcg} / \mathrm{l}$ using neoplastin plus compared with $700 \mathrm{mcg} / \mathrm{l}$ using Innovin (Samama et al. 2010; Perzborn et al. 2005). A systematic review of 49 articles showed that the prolongation of PT depends on the plasma concentration of RXA, but that the correlation was weaker above $50-100 \mathrm{mcg} / \mathrm{l}$ (Samuelson et al. 2017).

Although a significantly elevated INR was noted in patients taking DOAC (Ofek et al. 2017), conversion of PT 


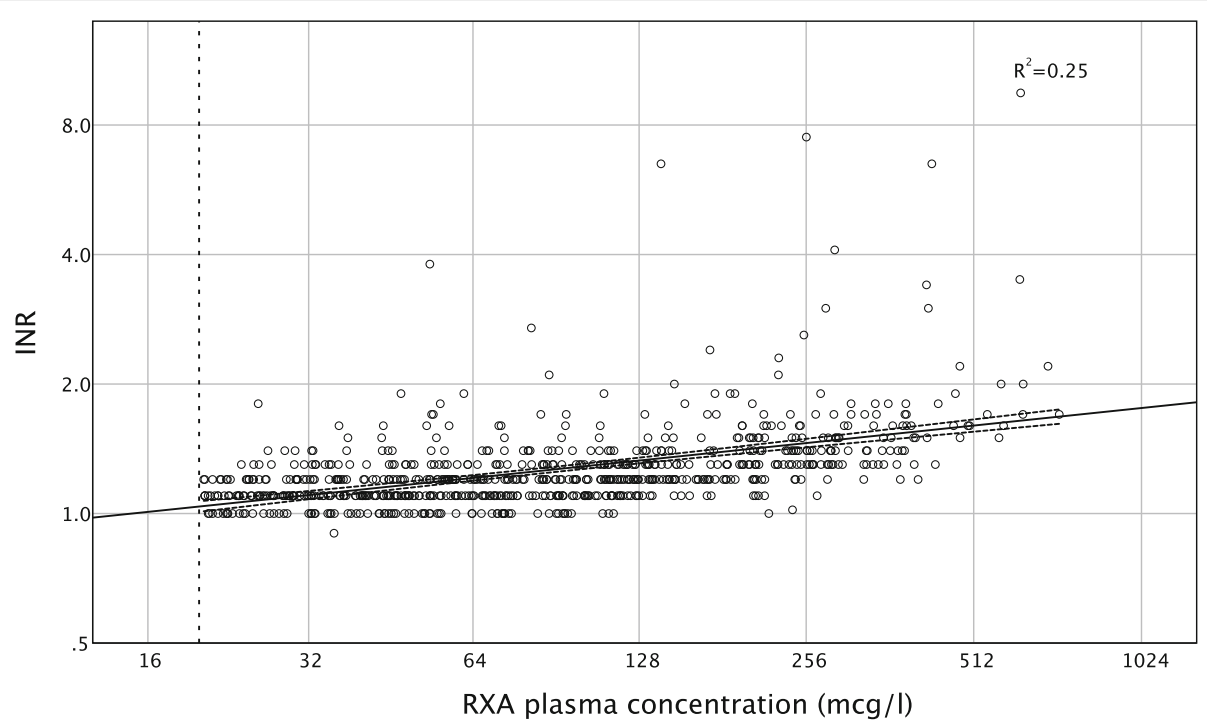

Fig. 3 Correlation of rivaroxaban plasma concentration and INR. A statistically significant correlation is observed (Pearson's correlation coefficient $0.5, p<0.001 ; R^{2}$ linear 0.25$)$. INR = international normalized ratio, $\mathrm{RXA}=$ rivaroxaban

to INR increased the variability and resulted in reduced RXA responsiveness (Siegal and Konkle 2014). This is not surprising since INR was designed for the monitoring of anticoagulation with vitamin $\mathrm{K}$ antagonists, with a focus on the usual target range of INR 2.0-3.0. Interestingly, only $25 \%$ of our patients with a normal INR $(<1.2)$ had a RXA plasma concentration above $50 \mathrm{mcg} / \mathrm{l}$ which is the lowest proportion compared to patients with normal aPTT and/or PT/Quick.

In line with the findings of our study, the Guidance from the British Committee for Standards in Haematology by Kitchen et al. concluded that PT and aPTT cannot be used to quantify RXA plasma concentration. At most, these assays - with some but not all reagents - may permit a crude estimation of the intensity of anticoagulation (Kitchen et al. 2014). AntiXa chromogenic assays should be used instead to determine RXA plasma concentration (Kitchen et al. 2014; Adcock and Gosselin 2015).

\section{Limitations}

Data of our study were collected retrospectively. Nevertheless, documentation and data collection followed

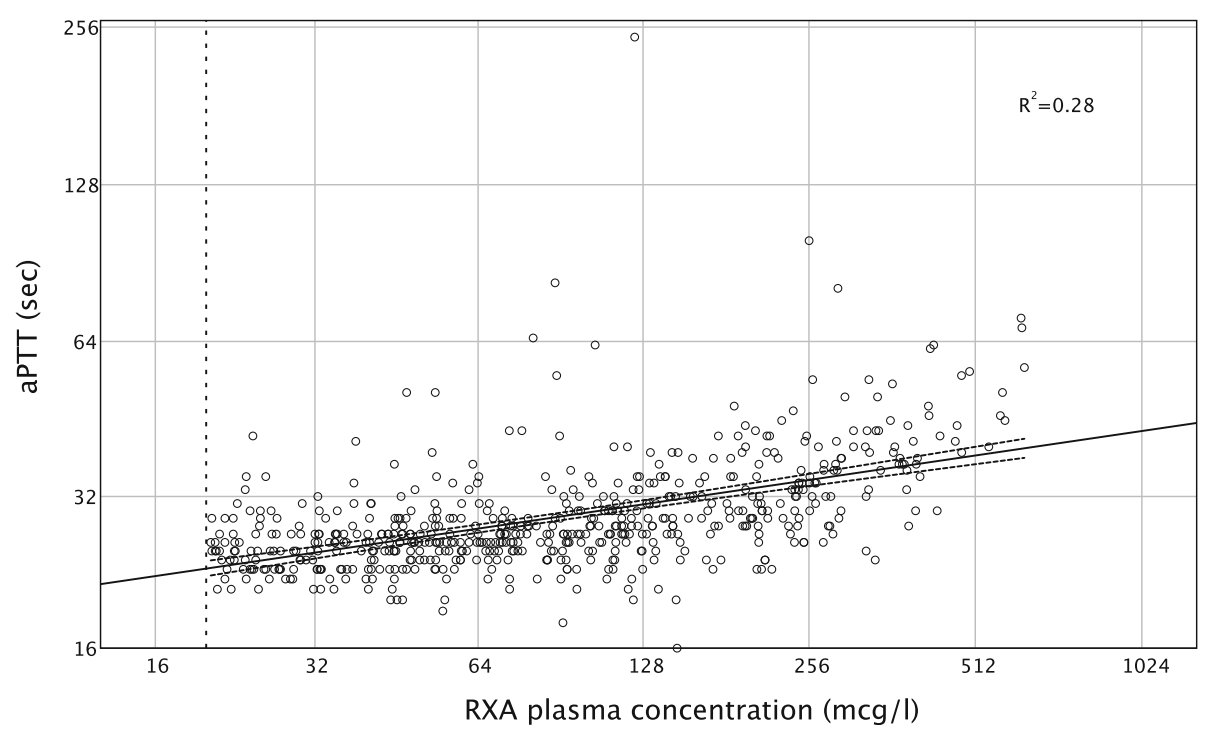

Fig. 4 Correlation of rivaroxaban plasma concentration and aPTT. A statistically significant correlation is observed (Pearson's correlation coefficient $0.53, p<0.001 ; R^{2}$ linear 0.28). aPTT = activated partial thromboplastin time, RXA = rivaroxaban 
Good Clinical Practice guidelines, and we assume that the data quality is high.

\section{Conclusion}

Although a moderate correlation of RXA plasma concentration with PT/Quick, INR and aPTT was observed, standard coagulation assays alone are not sufficient to exclude a surgically relevant RXA plasma concentration. Chromogenic anti-Xa assays should be used in patients with suspected RXA intake.

\begin{abstract}
Abbreviations
aPTT: Activated partial thromboplastin time; AUC: Area under the concentration-time curve; BMI: Body mass index; DOAC: Direct oral anticoagulants; GFR: Estimated glomerular filtration rate according to the CKD-EPI formula; INR: International normalized ratio; PT: Prothrombin time; ROC: Receiver operating characteristic; RXA: Rivaroxaban
\end{abstract}

\section{Acknowledgements}

Not applicable.

\begin{abstract}
Authors' contributions
AK planned the study, contributed to data collection, data interpretation, and wrote the manuscript. AS acquired the data, contributed to data interpretation, and wrote the manuscript. BS contributed to analysis and data interpretation. DS planned the study, contributed to data interpretation, and corrected the manuscript. JS participated in study planning, data acquisition, data interpretation, writing, and review of the manuscript. PS contributed to study planning, data interpretation and corrected the manuscript. All authors read and approved the final manuscript.
\end{abstract}

\section{Funding}

None.

\section{Availability of data and materials}

All data generated or analysed during this study are included in this published article.

\section{Ethics approval and consent to participate}

This study was approved by the local ethics committee (Kantonale Ethikkommission Zurich, Switzerland, KEK-ZH-No: 2017-00164).

\section{Consent for publication}

Not applicable.

\section{Competing interests}

DS' academic department is receiving grant support from the Swiss National Science Foundation, Berne, Switzerland, the Ministry of Health (Gesundheitsdirektion) of the Canton of Zurich, Switzerland for Highly Specialized Medicine, the Swiss Society of Anesthesiology and Reanimation (SGAR), Berne, Switzerland, the Swiss Foundation for Anesthesia Research, Zurich, Switzerland, CSL Behring, Berne, Switzerland, Vifor SA, Villars-surGlâne, Switzerland. DS is co-chair of the ABC-Trauma Faculty, sponsored by unrestricted educational grants from Novo Nordisk Health Care AG, Zurich, Switzerland, CSL Behring GmbH, Marburg, Germany, LFB Biomédicaments, Courtaboeuf Cedex, France and Octapharma AG, Lachen, Switzerland. DS has received honoraria or travel support for consulting or lecturing from: Danube University of Krems, Austria, US Department of Defense, WA, USA, European Society of Anesthesiology, Brussels, BE, Korea, Korean Society for Patient Blood Management, Seoul, Korea, Korean Society of Anesthesiologists, Seoul, Baxter AG, Volketswil, Switzerland, Baxter S.p.A., Roma, Italy, Bayer AG, Zürich, Switzerland, Bayer Pharma AG, Berlin, Germany, B. Braun Melsungen AG, Melsungen, Germany, Boehringer Ingelheim GmbH, Basel, Switzerland, BristolMyers-Squibb, Rueil-Malmaison Cedex, France and Baar, Switzerland, CSL Behring GmbH, Hattersheim am Main, Germany and Berne, Switzerland, Celgene International II Sàrl, Couvet, Switzerland, Curacyte AG, Munich, Germany, Daiichi Sankyo AG, Thalwil, Switzerland, GlaxoSmithKline GmbH \& Co. KG, Hamburg, Germany, Haemonetics, Braintree, MA, USA,
Instrumentation Laboratory (Werfen), Bedford, MA, USA, LFB Biomédicaments, Courtaboeuf Cedex, France, Merck Sharp \& Dohme, Kenilworth, NJ, USA, Octapharma AG, Lachen, Switzerland, Organon AG, Pfäffikon/SZ, Switzerland, PAION Deutschland GmbH, Aachen, Germany, Pharmacosmos A/S, Holbaek, Denmark, Photonics Healthcare B.V., Utrecht, Netherlands, Roche Diagnostics International Ltd, Reinach, Switzerland, Roche Pharma AG, Reinach, Switzerland, Sarstedt AG \& Co., Sevelen, Switzerland and Nümbrecht, Germany Schering-Plough International, Inc., Kenilworth, NJ, USA, Tem International GmbH, Munich, Germany, Verum Diagnostica GmbH, Munich, Germany, Vifor Pharma, Munich, Germany, Vienna, Austria and Villars-surGlâne, Switzerland, Vifor (International) AG, St. Gallen.

JS received lecture honoraria and advisory honoraria from Baxter (Switzerland), Bayer (Switzerland), BMS Pfizer (Switzerland), BoehringerIngelheim (Switzerland), CSL Behring (Switzerland), Janssen-Cilag (Switzerland), Mitsubishi Pharma, Novo Nordisk (Switzerland), Octapharma (Switzerland), Siemens Healthineers (Switzerland). PS received honoraria for lecturing by Vifor Pharma (Munich, Germany). All other authors declare that they have no competing interests.

\section{Author details}

${ }^{1}$ Institute of Anaesthesiology, University and University Hospital Zurich, Raemistrasse 100, 8091 Zurich, Switzerland. 'Department of Biostatistics at Epidemiology, Biostatistics and Prevention Institute, University of Zurich, Hirschengraben 84, 8001 Zurich, Switzerland. ${ }^{3}$ Division of Medical Oncology and Haematology, University and University Hospital Zurich, Raemistrasse 100, 8091 Zurich, Switzerland.

Received: 3 July 2019 Accepted: 16 October 2019

Published online: 20 November 2019

References

Adcock DM, Gosselin R. Direct oral anticoagulants (DOACs) in the laboratory: 2015 Review. Thromb Res. 2015;136(1):7-12.

Asmis LM, Alberio L, Angelillo-Scherrer A, Korte W, Mendez A, Reber G, et al. Rivaroxaban: Quantification by anti-FXa assay and influence on coagulation tests: a study in 9 Swiss laboratories. Thromb Res. 2012;129(4):492-8.

Baglin T. The role of the laboratory in treatment with new oral anticoagulants. J Thromb Haemost. 2013;11(Suppl 1):122-8.

Dale BJ, Ginsberg JS, Johnston M, Hirsh J, Weitz JI, Eikelboom JW. Comparison of the effects of apixaban and rivaroxaban on prothrombin and activated partial thromboplastin times using various reagents. J Thromb Haemost. 2014;12(11):1810-5.

Hillarp A, Gustafsson KM, Faxalv L, Strandberg K, Baghaei F, Fagerberg Blixter I, et al. Effects of the oral, direct factor Xa inhibitor apixaban on routine coagulation assays and anti-FXa assays. J Thromb Haemost. 2014;12(9): 1545-53.

Ikeda K, Tachibana H. Clinical implication of monitoring rivaroxaban and apixaban by using anti-factor Xa assay in patients with non-valvular atrial fibrillation. J Arrhythm. 2016;32(1):42-50.

Kaserer A, Schedler A, Jetter A, Seifert B, Spahn DR, Stein P, et al. Risk factors for higher-than-expected residual rivaroxaban plasma concentrations in real-life patients. Thromb Haemost. 2018;118(5):808-17.

Kitchen S, Gray E, Mackie I, Baglin T, Makris M, Committee B. Measurement of non-coumarin anticoagulants and their effects on tests of haemostasis: Guidance from the British Committee for Standards in Haematology. Br J Haematol. 2014;166(6):830-41.

Ofek F, Bar Chaim S, Kronenfeld N, Ziv-Baran T, Berkovitch M. International normalized ratio is significantly elevated with rivaroxaban and apixaban drug therapies: a retrospective study. Clin Ther. 2017;39(5):1003-10.

Perzborn E, Strassburger J, Wilmen A, Pohlmann J, Roehrig S, Schlemmer KH, et al. In vitro and in vivo studies of the novel antithrombotic agent BAY 597939--an oral, direct Factor Xa inhibitor. J Thromb Haemost. 2005;3(3):514-21.

Samama MM, Contant G, Spiro TE, Perzborn E, Le Flem L, Guinet C, et al. Laboratory assessment of rivaroxaban: a review. Thrombosis J. 2013;11(1):11.

Samama MM, Martinoli JL, LeFlem L, Guinet C, Plu-Bureau G, Depasse F, et al. Assessment of laboratory assays to measure rivaroxaban--an oral, direct factor Xa inhibitor. Thromb Haemost. 2010;103(4):815-25.

Samuelson BT, Cuker A, Siegal DM, Crowther M, Garcia DA. Laboratory assessment of the anticoagulant activity of direct oral anticoagulants: a systematic review. Chest. 2017;151(1):127-38. 
Siegal DM, Konkle BA. What is the effect of rivaroxaban on routine coagulation tests? Hematology Am Soc Hematol Educ Program. 2014;2014(1):334-6.

Studt JD, Alberio L, Angelillo-Scherrer A, Asmis LM, Fontana P, Korte W, et al. Accuracy and consistency of anti-Xa activity measurement for determination of rivaroxaban plasma levels. J Thromb Haemost. 2017;15(8):1576-83.

Swissmedicinfo.ch. Product information Xarelto ${ }^{\oplus}$ http://www.swissmedicinfo.ch/ [Accessed 13 Oct 2017]. 2017

Turkoglu El. NOACs and routine coagulation assays. How to interpret? Int J Cardiovasc Acad. 2015;1 (2-3):41-2

\section{Publisher's Note}

Springer Nature remains neutral with regard to jurisdictional claims in published maps and institutional affiliations.

Ready to submit your research? Choose BMC and benefit from:

- fast, convenient online submission

- thorough peer review by experienced researchers in your field

- rapid publication on acceptance

- support for research data, including large and complex data types

- gold Open Access which fosters wider collaboration and increased citations

- maximum visibility for your research: over $100 \mathrm{M}$ website views per year

At $B M C$, research is always in progress.

Learn more biomedcentral.com/submissions 